\title{
SOIL TAXONOMY AND EVALUATION OF SOME NEWLY RECLAIMED AREAS ADJACENT TO THE NILE DELTA RIMS, EGYPT
}

\author{
Salah M. Abu El Enain, Shaban M. Abd El-Rasoul* and \\ Mohamed M. Mohamed
}

Soils and Water Dept., Fac. of Agric., Al-Azhar University, Cairo, Egypt

*Soils, Water and Environ. Res. Institute, Agric. Res. Center, Giza, Egypt

\section{ABSTRACT:}

This study was carried out to identify the main soil characteristics, and then soil classification and evaluation of some newly reclaimed areas adjacent to the east, west and south Nile Delta rims, Egypt. Such work is considered as a scientific fundamental base for a successful agricultural development of such areas. To achieve this target, the main soil physical and chemical characteristics as well as plant nutrients status of the different soil types developed on the studied areas were determined, with a carefully study through representative eleven soil profiles.

The obtained analytical data indicate that soil texture is dominated with medium (i.e., sandy clay loam) and relatively coarse grades (i.e., sandy, loamy sand and sandy loam), except some soils of El-Hussania and Tal El-Yahudia areas that are characterized by fine texture grade of clayey and sandy clay, respectively. That is true, since these soils are developed on different origins, i.e., the Nile alluvium and desert formations calcareous or siliceous in nature as well as under various environmental conditions. Also, the soils under investigation are mostly non-saline, except of some ones are suffering from slight to high saline conditions at ElHussania, El-Nubaria and Abu-Zaabal areas, which are located at the east, west and south Nile Delta rims, respectively. In addition, the available plant nutrients status in the studied soils was more attributed with the additions of organic manures and agro-management practices as well as the nature of soil sediments and locally prevailing environmental conditions.

Soil taxa of the studied soils could be categorized into three orders, i.e., Vertisols, Entisols and Aridisols, and six families, as follows:

i. Vertisols include one family of Typic Haplotorrerts, fine clayey, smectitic, thermic (i.e., soils developed on El-Hussania area).

ii. Entisols: include four families of Typic Torrifluvents, sandy, mixed, thermic (i.e., soils developed on El-Qurein and El-Salhia areas); Typic Torrifluvents, clayey, smectitic, thermic (i.e., soils developed on Tal El-Yahudia village); Typic Torriorthents, sandy, mixed, thermic (i.e., soils developed on El-Alikat ElBahariya village) and Typic Torripsamments, siliceous, thermic (i.e., soils developed on El-Sadat and Abu-Zaabal areas).

iii. Aridisols: include one family of Typic Haplocalcids, fine loamy, mixed, thermic (i.e., soils developed on El-Beheira and El-Nubaria areas).

The data of the current suitability of the studied soils for irrigated agriculture indicate the existing of three suitability classes, i.e., marginally (S3, soils of ElSadat, Alikat and Abu-Zaabal areas), moderately (S2, soils of El-Qurein, El-Salhia, El-Beheira, El-Nubaria and Tal El-Yahudia areas) and highly suitable (S1, some soils of El-Beheira area). Soils of El-Nubaria can be shifted from the current suitability class of S2 to the potential suitability one of S1, due to their soil limitations are able to correct, i.e., wetness and salinity/alkalinity conditions.

It is noteworthy to mention that the similarity of suitability classes recognized in the studied areas for both current and potential conditions are mainly attributed to most of the identified soil limitations can not able to correct. So, it

Fayoum J. Agric. Res. \& Dev., Vol.24, No.2, July, 2010 
could be recommended that the severity of soil texture (coarser in nature) can be corrected by application of organic and inorganic soil amendments as well as applying drip and sprinkler irrigation systems to sustain a soil moisture content at a favourable condition for grown plants and biological activity in the soil.

Key Words: Soils of the Nile Delta rims, soil taxonomy and soil evaluation for irrigated agriculture.

\section{INTRODUCTION:}

The yearly progressive increase of human pressure on our limited cultivated areas in the Nile Valley and Delta requires to pay a suitable attention to horizontal expansion, especially for the soils adjacent to such regions and to conserve their productivity status. Since the land potentiality for agricultural aspects is the most recognized problem threatens these soils, the Egyptian Government efforts have been directed towards both identifying soil productivity limitations and executing the suitable agro-management practices. Also, land potentiality for agricultural purposes is mainly attributed to the changes in the natural environmental conditions as well as the human agro-management practices of the natural resources, intensive cultivation by using suitable irrigation systems and farming practices (FAO, 1983).

A full understanding of soil physical and chemical characteristics as well as nutrients status of some promising areas at the east, west and south Nile Delta rims, Egypt is considered as the fundamental base for a successful agricultural development of these areas. Such aspect represents a pressing need that should be an urgent solves to set up expansion or amelioration programs out-side the limited area of agricultural land confining the Nile Valley and Delta as well as to overcome the fast growing population in Egypt. Identifying such database of soil characteristics will be useful as a guide for successful fertilization system, and in turn it is of vita importance for agricultural utilization programs as well as to get the highest agricultural productivity from these soils to overcome the nutritional security needs for human.

The majority of soils in arid and semi arid regions are desert sandy or calcareous in nature, having poor hydrophysical or fertility status and suffering from a shortage in fresh irrigation water resources (Gupta, 1990). Hence, this scientific work was carried out to identify the main soil characteristics, soil taxonomy and evaluation as affected by soil origins and the prevailing environmental conditions of east, west and south Nile Delta rims, Egypt.

\section{MATERIALS AND METHODS: \\ a. Field work:}

Representative eleven soil profiles were selected from the main soil types in the east, west and south Nile Delta rims, Map (1). Disturbed soil samples were obtained from each soil profile according to the morphological features throughout its layers by following the guidelines after FAO (1990), and then they were preparing to determine the main soil characteristics,

\section{b. Laboratory analyses:}

Particle size distribution was determined according to Gee and Bauder (1986), using sodium hexameta phosphate as dispersing agent Richards (1954). $\mathrm{CaCO}_{3}$ content was determined volumetrically by using Scheibler's Calcimeter (Black et al., 1965). Organic matter was determined using the Walkley-Black dichromate acid oxidation method according to Jackson (1973). Gypsum content was quantitatively determined using acetone method (Black et al., 1965).

Fayoum J. Agric. Res. \& Dev., Vol.24, No.2, July, 2010 


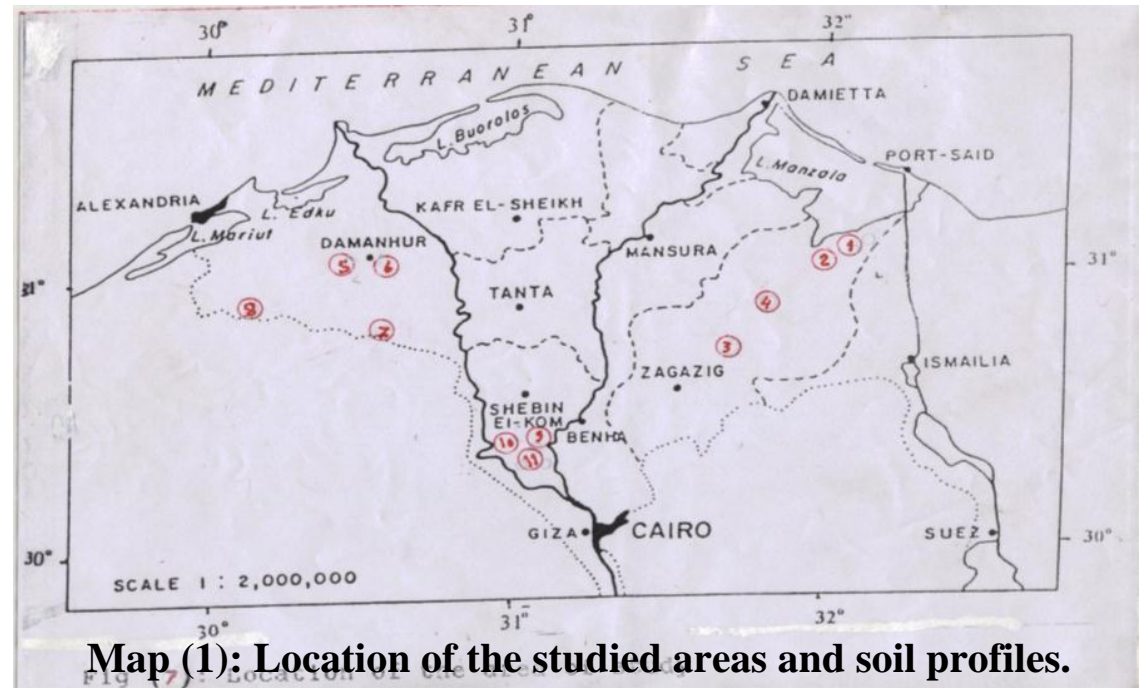

Soil $\mathrm{pH}$ was measured in 1:2.5 soil : water suspensions by using a $\mathrm{pH}$ meter according to Jackson (1973). Soil paste extract was prepared for determining electrical conductivity (ECe) and soluble ions according to Jackson (1967). Available macronutrients of $\mathrm{N}, \mathrm{P}$ and $\mathrm{K}$ in the soil were extracted by $1 \%$ potassium sulphate, $0.5 \mathrm{M}$ sodium bicarbonate and $1 \mathrm{~N}$ ammonium acetate, respectively (Soltanpour and Schwab, 1977), and then their contents were determined according to Jackson (1973). Available micronutrients of Fe, Mn, Zn, and $\mathrm{Cu}$ in soil were extracted using diethylene triamine penta acetic acid (DTPA) by a solution consisting of $0.005 \mathrm{M}$ DTPA, $0.01 \mathrm{M} \mathrm{CaCl}_{2}$ and $0.1 \mathrm{M}$ TEA (triethanol-amine) with the $\mathrm{pH}$ adjusted to 7.3 by hydrochloric acid $(1: 1)$ according to Follet and Lindsay (1971) and Lindsay and Norvell (1978), and are determined by using Atomic Absorption Spectro-photometer (model GBC 932).

\section{c. Soil classification:}

Soil Taxa for the studied areas were identified according to USDA (1975 and 2006), which based on matching the morphological features with the main analytical data of the soils under investigation.

d. Land evaluation for irrigated agriculture:

Land evaluation for irrigated agriculture was estimated by matching between the present soil characteristics and their ratings by using the parametric system outlined by Sys and Verheye (1978) and Sys (1991), considering the soil limitation intensities.

\section{RESULTS AND DISCUSSION:}

\section{A general view on the investigated soils:}

The studied soils are developed on different origins, i.e., the Nile alluvium (soil profile Nos. 1 and 2), fluvio-aeolian deposits (soil profile Nos. 3, 4, 9 and 10), Miocene limestone deposits (soil profile Nos. 5, 6 and 8) and the deserted wind blown sand or aeolian deposits (soil profile Nos. 7 and 11). The soil texture throughout the entire soil profile depths is either uniform (i.e., soil profile Nos. 1, 2, 5, 7 and 11), or variable (i.e., soil profile Nos. 3, 4, 6, 8, 9 and 10) according to the nature of soil sediment origins and depositional regime. The studied soils showed a weak evidence of soil development, except of some soil sites of ElBeheira and Nubaria (i.e., soil profile Nos. 5, 6 and 8), however, secondary

Fayoum J. Agric. Res. \& Dev., Vol.24, No.2, July, 2010 
formations of $\mathrm{CaCO}_{3}$ vary widely from few to many in forms of soft fine crystals, calcic nodules or segregations and concretions, which its content is enough to identify a calcic horizon. It is noteworthy to mention that the accumulation of salts in some soil sites, i.e., El-Hussania (high and slight saline of soil profile Nos. 1 and 2, respectively), El-Nubaria and Abu-Zaabal (moderate saline of soil profile Nos. 8 and 11). In general, the occurrence of these secondary formations (i.e., $\mathrm{CaCO}_{3}$ and salts) throughout the soil profile layers reflects to a great extent, its formation mode under the prevailing arid conditions.

\section{Physical and chemical properties of the studied soils:}

a. Particle size distribution, $\mathrm{CaCO}_{3}$, organic matter and gypsum contents:

Exception of El-Hussania (clayey) and Tal El-Yahudia (sandy clay) soil sites that are characterized by fine texture grade, the obtained data in Table (1) show that soil texture is dominated with medium, i.e., sandy clay loam of soil profile Nos. 5 and 8, besides the relatively coarse grades, i.e., sand of soil profile Nos. 7 and 11, loamy sand and sandy loam of soil profile Nos. 3, 4, 6 and 10. That is true, since the studied soils are developed on different origins (i.e., the Nile alluvium and desert formations calcareous or siliceous in nature) and under various locally environmental conditions.

The obtained data of calcium carbonate and organic matter contents are shown in Table (1). These data show that the distribution of $\mathrm{CaCO}_{3}$ in the studied soil profiles is greatly varied from 0.32 to $43.41 \%$ (deepest profile layers of ElQurein and El-Nubaria, respectively). In general, the greatest and lowest $\mathrm{CaCO}_{3}$ contents are more related to the desert formations either calcareous (Miocene limestone) or siliceous (aeolian deposits) in nature, respectively. The distribution pattern of $\mathrm{CaCO}_{3}$ tends to increase with depth as shown in soil profile Nos. 2, 4 and 10, while the reverse is true for soil profile Nos. 1, 3, 7 and 11. In addition, $\mathrm{CaCO}_{3}$ content exhibited an irregular pattern throughout soil profile layers Nos. 5, 6, 8 and 9. Gypsum content is never exceeding $1.0 \%$ of soil components, where its values ranged 0.07-0.97\%. Exception of soil profile Nos. 6 and 9 that showed an irregular distribution pattern of gypsum throughout soil profile layers, the soils of the other studied sites exhibited a tendency decrease for gypsum content with depth. Such different distribution patterns of either $\mathrm{CaCO}_{3}$ or gypsum content may be attributed to the different sedimentation cycles of the soil deposits and partly to chemical weathering intensity and their mobility either up- or downmovement within the entire soil profile.

Data in Table (1) show also that organic matter content is relatively low, never exceeding $1.62 \%$ of soil components, probably due to the high oxidation potential sustained under the prevailing arid and hot climate as well as insufficient organic fertilization. It is clear that the relatively higher values of organic matter content present in the surface layer of soil profile, and then it tends to decrease with depth. The values of soil organic matter content ranged from 0.05 (deepest layer of Abu-Zaabal soil) to $1.62 \%$ (surface layer of El-Qurein soil). As expected, the relatively high content in the surface layers of these soils are associated with the agro-management or cultivation practices. It is noteworthy to mention that the only organic matter source in some desert barren soils is more attributed to the plant residues of some native vegetation.

\section{b. Soil $\mathrm{pH}$ and salinity:}

As shown in Table (2), soil $\mathrm{pH}$ values are found at a nearly neutral phase, except of soil profile No. 4 that tends to alkaline side (soil $\mathrm{pH}$ of 8.55). In general, soil $\mathrm{pH}$ value tends to increase with depth as shown in soil profile Nos. 1, 2, 4, 6, 9, 10 and 11 , while the reverse was true for soil profile Nos. 3, 5, 7 and 8 .

Fayoum J. Agric. Res. \& Dev., Vol.24, No.2, July, 2010 
SOIL TAXONOMY AND EVALUATION OF SOME NEWLY.

Table (1): Particle size distribution, $\mathrm{CaCO}_{3}$, gypsum and organic matter contents of the representative soil sites.

\begin{tabular}{|c|c|c|c|c|c|c|c|c|c|c|c|}
\hline \multicolumn{2}{|c|}{ Location } & \multirow{2}{*}{ 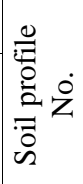 } & \multirow[b]{2}{*}{$\begin{array}{l}\text { Depth } \\
(\mathrm{cm})\end{array}$} & \multicolumn{4}{|c|}{ Particle size distribution $\%$} & \multirow{2}{*}{ 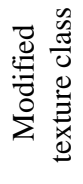 } & \multirow{2}{*}{$\begin{array}{l}\delta^{0} \\
0_{\tilde{J}}^{m} \\
0\end{array}$} & \multirow[b]{2}{*}{ 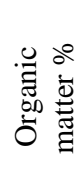 } & \multirow{2}{*}{ 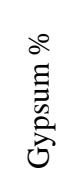 } \\
\hline $\begin{array}{l}.0 \\
.00 \\
\mathscr{0} \\
\simeq\end{array}$ & 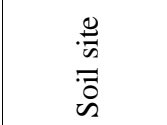 & & & 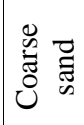 & 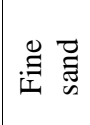 & 责 & $\frac{\vec{\Xi}}{U}$ & & & & \\
\hline \multirow{12}{*}{ 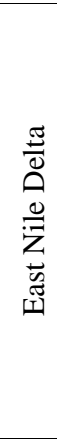 } & \multirow{6}{*}{ 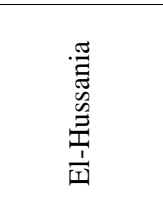 } & & $0-25$ & 12.00 & 6.80 & 38.05 & 43.15 & \multirow{6}{*}{$\mathrm{C}$} & 4.62 & 0.46 & 0.67 \\
\hline & & 1 & $25-60$ & 13.50 & 4.67 & 36.33 & 45.50 & & 3.21 & 0.24 & 0.55 \\
\hline & & & $60-90$ & 2.64 & 4.25 & 38.61 & 54.50 & & 2.01 & 0.13 & 0.45 \\
\hline & & & $0-30$ & 1.76 & 8.50 & 39.70 & 50.04 & & 3.20 & 0.33 & 0.85 \\
\hline & & 2 & $30-60$ & 2.30 & 13.60 & 36.10 & 48.00 & & 3.63 & 0.15 & 0.76 \\
\hline & & & $60-100$ & 4.35 & 11.70 & 38.84 & 45.11 & & 3.72 & 0.09 & 0.49 \\
\hline & \multirow{3}{*}{$\begin{array}{c}\text { El- } \\
\text { Qurein }\end{array}$} & \multirow{3}{*}{3} & $0-30$ & 53.80 & 19.0 & 13.0 & 8.80 & \multirow{3}{*}{$\begin{array}{c}\text { SL } \\
\text { SCL } \\
\text { LS }\end{array}$} & 1.67 & 1.62 & 0.47 \\
\hline & & & $30-60$ & 34.90 & 26.8 & 11.20 & 22.70 & & 0.43 & 0.36 & 0.32 \\
\hline & & & $60-90$ & 70.30 & 12.9 & 6.30 & 9.00 & & 0.32 & 0.25 & 0.28 \\
\hline & \multirow{3}{*}{$\begin{array}{l}\text { El- } \\
\text { Salhia }\end{array}$} & \multirow{3}{*}{4} & $0-30$ & 44.65 & 10.98 & 20.15 & 24.22 & \multirow{3}{*}{$\begin{array}{c}\text { SCL } \\
\text { SL } \\
\text { LS }\end{array}$} & 6.86 & 1.45 & 0.70 \\
\hline & & & $30-60$ & 48.20 & 10.44 & 24.87 & 16.49 & & 8.24 & 1.47 & 0.52 \\
\hline & & & $60-90$ & 63.30 & 15.73 & 13.47 & 7.50 & & 11.05 & 1.56 & 0.37 \\
\hline \multirow{13}{*}{$\begin{array}{l}\frac{\pi}{0} \\
0 \\
\frac{0}{0} \\
\bar{Z} \\
\overrightarrow{0} \\
\frac{0}{3}\end{array}$} & \multirow{3}{*}{$\begin{array}{c}\text { El- } \\
\text { Beheira }\end{array}$} & \multirow{3}{*}{5} & 0.30 & 16.45 & 61.35 & 2.20 & 20.0 & \multirow{3}{*}{ SCL } & 24.55 & 1.28 & 0.94 \\
\hline & & & $30-70$ & 12.23 & 37.50 & 18.28 & 29.00 & & 34.30 & 0.45 & 0.90 \\
\hline & & & $70-150$ & 14.36 & 42.27 & 17.1 & 25.0 & & 23.00 & 0.11 & 0.86 \\
\hline & \multirow{4}{*}{$\begin{array}{c}\text { El- } \\
\text { Beheira }\end{array}$} & \multirow{4}{*}{6} & $0-30$ & 15.01 & 46.62 & 22.38 & 16.0 & SL & 23.60 & 1.29 & 0.52 \\
\hline & & & $30-60$ & 13.94 & 54.31 & 14.75 & 17.0 & SL & 30.76 & 0.70 & 0.64 \\
\hline & & & $60-90$ & 15.69 & 44.66 & 17.65 & 22.0 & SCL & 26.07 & 0.62 & 0.35 \\
\hline & & & $90-150$ & 16.45 & 43.55 & 20.00 & 20.0 & SCL & 22.53 & 0.53 & 0.41 \\
\hline & \multirow{3}{*}{$\begin{array}{c}\text { El- } \\
\text { Sadat }\end{array}$} & \multirow{3}{*}{7} & $0-50$ & 45.30 & 50.00 & 3.20 & 2.50 & & 2.21 & 1.40 & 0.24 \\
\hline & & & $50-110$ & 40.00 & 57.30 & 1.60 & 1.10 & $\mathrm{~S}$ & 1.38 & 1.02 & 0.17 \\
\hline & & & $110-150$ & 44.00 & 50.40 & 3.30 & 2.10 & & 1.04 & 1.08 & 0.09 \\
\hline & & & $0-25$ & 25.06 & 40.07 & 20.88 & 14.00 & $\mathrm{SL}$ & 32.67 & 0.37 & 0.97 \\
\hline & $\begin{array}{c}\text { El- } \\
\text { Nubaria }\end{array}$ & 8 & $25-75$ & 60.22 & 14.03 & 2.63 & 23.13 & SCL & 24.81 & 0.20 & 0.88 \\
\hline & & & $75-90$ & 36.74 & 19.24 & 18.15 & 25.85 & SCL & 43.41 & 0.12 & 0.75 \\
\hline & & & $0-30$ & 46.68 & 15.23 & 9.52 & 28.57 & SCL & 2.62 & 0.36 & 0.62 \\
\hline & $\begin{array}{l}\text { 1 al El- } \\
\text { Yahudia }\end{array}$ & 9 & $30-75$ & 36.12 & 9.97 & 12.24 & 41.67 & $\mathrm{SC}$ & 3.25 & 0.20 & 0.74 \\
\hline & $\begin{array}{l}\text { Yanudia } \\
\text { village }\end{array}$ & 9 & $75-110$ & 39.08 & 8.09 & 12.20 & 40.63 & SC & 4.01 & 0.13 & 0.90 \\
\hline อั & & & $110-150$ & 28.80 & 7.46 & 15.58 & 48.16 & $\mathrm{C}$ & 1.54 & 0.47 & 0.87 \\
\hline$\stackrel{0}{=}$ & El-Alikat & 10 & 0-30 & 57.60 & 14.30 & 6.61 & 21.49 & SCL & 2.20 & 0.40 & 0.45 \\
\hline $\bar{Z}$ & El-Bahariya & 10 & $30-90$ & 80.01 & 9.74 & 2.77 & 7.48 & $\mathrm{~S}$ & 4.20 & 0.12 & 0.15 \\
\hline Ẽ & & & $0-40$ & 75.80 & 18.78 & 0.25 & 5.17 & & 5.10 & 0.12 & 0.34 \\
\hline ¿̊s & & & $40-70$ & 72.98 & 21.02 & 0.73 & 5.27 & & 4.60 & 0.11 & 0.21 \\
\hline & Abu-Zaabal & 11 & $70-100$ & 82.40 & 10.85 & 0.52 & 6.23 & $\mathrm{~S}$ & 4.00 & 0.09 & 0.13 \\
\hline & & & $100-150$ & 82.68 & 11.16 & 0.98 & 5.18 & & 3.90 & 0.05 & 0.07 \\
\hline
\end{tabular}

$\mathrm{S}=$ Sand, $\mathrm{LS}=$ Loamy sand, $\mathrm{SL}=$ Sandy loam, $\mathrm{SCL}=$ Sandy clay loam, $\mathrm{SC}=$ Sandy clay, $\mathrm{C}=$ Clay.

Soil salinity represents a prevailing phenomenon feature that mostly occurs in the arid and semi regions due to scarce rainfall and relatively high temperature of hot climate, which in turn resulted in the built up and accumulation of soluble salts. So that, the irrigated agriculture soils under the arid and semi arid zones are suffering from the process of soil salinization, which is more often connected with a shallow saline water table and high evaporation rate that are occurred among some investigated soil sites. Data presented in Table (2) show that the amount of total soluble salts as expressed by the electrical conductivity values (ECe in $\mathrm{dSm}^{-1}$ ) of the soil saturation extract ranged between 0.65 and $30.00 \mathrm{dSm}^{-1}$ as shown in soil profile Nos. 3 and 1, respectively. Also, sodium is the dominant soluble cation, however, the soluble cations could be arranged in the following

Fayoum J. Agric. Res. \& Dev., Vol.24, No.2, July, 2010 
Salah M. Abu El Enain, et al.

descending order of $\mathrm{Na}^{+}>\mathrm{Mg}^{2+}>\mathrm{Ca}^{2+}>\mathrm{K}^{+}$. Chloride is also the dominant soluble anion and the soluble anions could be arranged as follows: $\mathrm{Cl}^{-}>\mathrm{SO}_{4}{ }^{2-}>$ $\mathrm{HCO}_{3}{ }^{-}$in the different soil profile layers.

Table (2): Chemical analysis of soil paste extract of the studied soil profiles.

\begin{tabular}{|c|c|c|c|c|c|c|c|c|c|c|c|}
\hline \multirow{2}{*}{$\begin{array}{c}\text { Profile } \\
\text { No. }\end{array}$} & \multirow{2}{*}{$\begin{array}{l}\text { Depth } \\
(\mathrm{cm})\end{array}$} & \multirow{2}{*}{$\begin{array}{c}\text { Soil } \\
\mathrm{pH}\end{array}$} & \multirow{2}{*}{$\begin{array}{c}\mathrm{ECe} \\
(\mathrm{dS} / \mathrm{m})\end{array}$} & \multicolumn{4}{|c|}{ Soluble cations $\left(\mathrm{m}\right.$ mmolc $\left.\mathrm{L}^{-1}\right)$} & \multicolumn{4}{|c|}{ Soluble anions $\left(\mathrm{m}\right.$ mmolc L $\left.{ }^{-1}\right)$} \\
\hline & & & & $\mathrm{Ca}^{2+}$ & $\mathrm{Mg}^{2+}$ & $\mathrm{Na}^{+}$ & $\mathrm{K}^{+}$ & $\mathrm{CO}_{3}{ }^{2-}$ & $\mathrm{HCO}_{3}{ }^{-}$ & $\mathrm{Cl}^{-}$ & $\mathrm{SO}_{4}{ }^{2-}$ \\
\hline \multirow{3}{*}{1} & $0-25$ & 7.50 & 30.00 & 49.30 & 47.20 & 200.50 & 3.00 & 0.00 & 2.40 & 200.40 & 97.20 \\
\hline & $25-60$ & 7.75 & 24.00 & 11.70 & 58.70 & 166.80 & 2.74 & 0.00 & 2.14 & 204.66 & 33.14 \\
\hline & $60-90$ & 7.65 & 20.40 & 11.70 & 35.60 & 154.10 & 2.54 & 0.00 & 3.20 & 153.60 & 47.14 \\
\hline \multirow{3}{*}{2} & $0-30$ & 7.60 & 6.50 & 9.50 & 20.10 & 33.71 & 1.69 & 0.00 & 3.20 & 11.52 & 50.28 \\
\hline & $30-60$ & 7.75 & 7.90 & 15.40 & 24.90 & 36.53 & 2.17 & 0.00 & 2.40 & 22.90 & 53.70 \\
\hline & $60-100$ & 7.80 & 9.10 & 15.70 & 25.10 & 47.40 & 2.80 & 0.00 & 2.14 & 26.70 & 62.16 \\
\hline \multirow{3}{*}{3} & $0-30$ & 7.80 & 0.95 & 2.82 & 4.14 & 2.61 & 0.23 & 0.00 & 5.42 & 1.22 & 2.91 \\
\hline & $30-60$ & 7.50 & 0.65 & 2.16 & 1.88 & 2.50 & 0.19 & 0.00 & 2.08 & 2.09 & 3.80 \\
\hline & $60-90$ & 7.10 & 0.95 & 1.97 & 2.17 & 4.80 & 0.18 & 0.00 & 2.91 & 2.59 & 3.77 \\
\hline \multirow{3}{*}{4} & $0-30$ & 8.35 & 3.00 & 12.24 & 5.00 & 12.40 & 0.36 & 0.00 & 2.81 & 17.76 & 9.43 \\
\hline & $30-60$ & 8.50 & 2.30 & 8.46 & 4.55 & 9.64 & 0.35 & 0.00 & 2.74 & 11.76 & 8.50 \\
\hline & $60-90$ & 8.55 & 1.85 & 8.97 & 3.61 & 5.57 & 0.35 & 0.00 & 2.20 & 8.28 & 8.20 \\
\hline \multirow{3}{*}{5} & 0.30 & 8.30 & 2.88 & 2.64 & 2.48 & 14.00 & 0.52 & 0.00 & 3.20 & 4.80 & 11.64 \\
\hline & $30-70$ & 8.00 & 2.12 & 3.08 & 8.84 & 7.72 & 0.20 & 0.00 & 3.40 & 11.04 & 6.46 \\
\hline & $70-150$ & 8.10 & 1.92 & 2.64 & 4.58 & 7.50 & 0.32 & 0.00 & 3.60 & 6.92 & 4.52 \\
\hline \multirow{4}{*}{6} & $0-30$ & 8.10 & 3.04 & 1.00 & 3.28 & 18.32 & 0.24 & 0.00 & 3.00 & 3.12 & 16.72 \\
\hline & $30-60$ & 8.12 & 3.92 & 3.06 & 7.16 & 19.32 & 0.36 & 0.00 & 4.60 & 8.84 & 16.46 \\
\hline & $60-90$ & 8.40 & 3.88 & 2.16 & 4.64 & 20.24 & 0.32 & 0.00 & 4.80 & 2.80 & 21.76 \\
\hline & $90-150$ & 8.50 & 3.80 & 3.08 & 1.2 & 19.08 & 0.24 & 0.00 & 4.00 & 2.12 & 17.48 \\
\hline \multirow{3}{*}{7} & $0-50$ & 8.10 & 1.34 & 1.76 & 4.64 & 4.76 & 2.20 & 0.00 & 2.00 & 2.12 & 9.24 \\
\hline & $50-110$ & 8.10 & 1.43 & 2.20 & 3.76 & 6.76 & 1.24 & 0.00 & 1.60 & 2.12 & 10.24 \\
\hline & $110-150$ & 8.00 & 2.64 & 4.32 & 3.38 & 7.08 & 1.32 & 0.00 & 2.80 & 8.60 & 4.70 \\
\hline \multirow{3}{*}{8} & $0-25$ & 8.40 & 14.8 & 21.04 & 28.36 & 5.32 & 0.40 & 0.00 & 3.60 & 27.16 & 24.36 \\
\hline & $25-75$ & 8.20 & 6.60 & 33.80 & 22.96 & 4.24 & 0.36 & 0.00 & 3.40 & 30.20 & 27.76 \\
\hline & $75-90$ & 8.02 & 4.52 & 11.76 & 7.64 & 20.88 & 0.32 & 0.00 & 3.80 & 6.28 & 9.20 \\
\hline \multirow{4}{*}{9} & $0-30$ & 7.50 & 1.21 & 4.17 & 1.47 & 5.70 & 0.38 & 0.00 & 3.64 & 2.94 & 5.14 \\
\hline & $30-75$ & 7.75 & 1.26 & 3.65 & 2.51 & 5.70 & 0.11 & 0.00 & 2.60 & 2.94 & 6.43 \\
\hline & $75-110$ & 7.65 & 1.41 & 3.65 & 2.51 & 7.43 & 0.14 & 0.00 & 2.08 & 2.95 & 8.71 \\
\hline & $110-150$ & 7.70 & 1.31 & 2.61 & 2.52 & 7.20 & 0.12 & 0.00 & 2.08 & 2.94 & 7.43 \\
\hline \multirow{2}{*}{10} & $0-30$ & 8.00 & 5.86 & 13.55 & 10.05 & 32.40 & 1.94 & 0.00 & 3.20 & 30.42 & 24.32 \\
\hline & $30-90$ & 8.10 & 4.45 & 10.42 & 8.05 & 24.15 & 0.85 & 0.00 & 2.60 & 21.56 & 19.31 \\
\hline \multirow{4}{*}{11} & $0-40$ & 7.50 & 9.90 & 25.01 & 2.69 & 66.23 & 0.82 & 0.00 & 2.08 & 15.68 & 76.99 \\
\hline & $40-70$ & 7.75 & 9.70 & 26.01 & 5.77 & 62.60 & 0.28 & 0.00 & 2.08 & 13.72 & 78.86 \\
\hline & $70-100$ & 7.80 & 5.16 & 18.76 & 6.89 & 23.20 & 0.70 & 0.00 & 2.60 & 7.84 & 39.11 \\
\hline & $100-150$ & 8.00 & 1.30 & 3.65 & 1.48 & 7.45 & 0.12 & 0.00 & 2.60 & 1.96 & 8.14 \\
\hline
\end{tabular}

III. Available macro- and micro-nutrients status:

\section{a. Macronutrients:}

Data presented in Table (3) show that the values of chemically extractable $\mathrm{N}$ ranged between 10 and $42 \mathrm{mg} \mathrm{kg}^{-1}$, however, the highest and lowest values are found in the surface and deepest layers of soil profile Nos. 1 (soils of ElHussaania) and 11 (soils of Abu-Zaabal), respectively. Available phosphorus and potassium showed a similar trend of available $\mathrm{N}$ either in the studied soil sites or profile layers, where their values ranged $1.20-5.25$ and $48-415 \mathrm{mg} \mathrm{kg} \mathrm{kg}^{-1}$, respectively. In general, the vertical distribution of available $\mathrm{N}$ and $\mathrm{P}$ contents showed a tendency to accumulate in the surface layers. This behavior may be due to the applied organic fertilization and agro-management practices as well as the nature of soil sediments. 
Table (3): Available nutrient contents of the representative soil sites.

\begin{tabular}{|c|c|c|c|c|c|c|c|c|}
\hline \multirow{2}{*}{$\begin{array}{l}\text { Soil } \\
\text { site } \\
\text { No. }\end{array}$} & \multirow{2}{*}{$\begin{array}{c}\text { Depth } \\
(\mathrm{cm})\end{array}$} & \multicolumn{3}{|c|}{ Macronutrients $\left(\mathrm{mg} \mathrm{kg}^{-1}\right)$} & \multicolumn{4}{|c|}{ Micronutrients (mg kg ${ }^{-1}$ ) } \\
\hline & & $\mathrm{N}$ & $\mathrm{P}$ & $\mathrm{K}$ & $\mathrm{Fe}$ & $\mathrm{Mn}$ & $\mathrm{Zn}$ & $\mathrm{Cu}$ \\
\hline \multirow{3}{*}{1} & $0-25$ & 42.00 & 5.25 & 415 & 6.94 & 2.14 & 1.89 & 1.60 \\
\hline & $25-60$ & 30.30 & 4.40 & 401 & 5.68 & 1.92 & 1.32 & 1.08 \\
\hline & $60-90$ & 25.00 & 3.63 & 377 & 4.94 & 1.05 & 1.12 & 1.04 \\
\hline \multirow{3}{*}{2} & $0-30$ & 18.90 & 4.20 & 528 & 3.10 & 1.80 & 1.76 & 1.28 \\
\hline & $30-60$ & 17.50 & 3.40 & 519 & 1.68 & 1.60 & 1.60 & 1.07 \\
\hline & $60-100$ & 17.50 & 3.40 & 479 & 1.54 & 1.40 & 0.92 & 0.86 \\
\hline \multirow{3}{*}{3} & $0-30$ & 30.00 & 3.00 & 200 & 3.05 & 2.00 & 1.50 & 1.00 \\
\hline & $30-60$ & 15.00 & 3.22 & 195 & 4.45 & 1.95 & 1.04 & 0.89 \\
\hline & $60-90$ & 20.00 & 2.85 & 201 & 2.65 & 1.85 & 0.94 & 0.78 \\
\hline \multirow{3}{*}{4} & $0-30$ & 24.50 & 5.00 & 156 & 4.08 & 0.90 & 0.93 & 0.82 \\
\hline & $30-60$ & 19.80 & 3.90 & 123 & 2.36 & 0.79 & 0.74 & 0.64 \\
\hline & $60-90$ & 18.00 & 3.80 & 95 & 1.90 & 0.65 & 0.58 & 0.46 \\
\hline \multirow{3}{*}{5} & 0.30 & 48.00 & 4.20 & 220 & 4.50 & 1.64 & 1.08 & 0.97 \\
\hline & $30-70$ & 35.00 & 3.30 & 200 & 3.02 & 1.28 & 0.94 & 0.82 \\
\hline & $70-150$ & 35.00 & 3.00 & 200 & 2.26 & 0.95 & 0.81 & 0.75 \\
\hline \multirow{4}{*}{6} & $0-30$ & 30.00 & 3.50 & 180 & 5.24 & 2.75 & 1.16 & 0.98 \\
\hline & $30-60$ & 37.00 & 3.20 & 200 & 4.82 & 1.21 & 0.96 & 0.85 \\
\hline & $60-90$ & 37.00 & 3.40 & 195 & 3.26 & 0.99 & 0.85 & 0.63 \\
\hline & $90-150$ & 20.00 & 2.50 & 156 & 2.42 & 0.64 & 0.76 & 0.52 \\
\hline \multirow{3}{*}{7} & $0-50$ & 35.00 & 2.90 & 70 & 3.90 & 0.80 & 0.89 & 0.81 \\
\hline & $50-110$ & 40.00 & 2.65 & 81 & 2.56 & 0.60 & 0.78 & 0.54 \\
\hline & $110-150$ & 25.00 & 2.00 & 65 & 3.10 & 0.45 & 0.76 & 0.43 \\
\hline \multirow{3}{*}{8} & $0-25$ & 20.30 & 2.20 & 100 & 4.20 & 0.85 & 1.06 & 0.85 \\
\hline & $25-75$ & 19.10 & 1.70 & 79 & 3.60 & 0.72 & 0.95 & 0.67 \\
\hline & $75-90$ & 15.00 & 1.40 & 80 & 2.24 & 0.80 & 0.87 & 0.54 \\
\hline \multirow{4}{*}{9} & $0-30$ & 32.00 & 3.25 & 200 & 7.54 & 1.25 & 1.10 & 0.89 \\
\hline & $30-75$ & 20.30 & 2.40 & 395 & 5.15 & 1.15 & 1.08 & 0.75 \\
\hline & $75-110$ & 25.00 & 2.43 & 477 & 4.89 & 0.95 & 0.94 & 0.65 \\
\hline & $110-150$ & 20.00 & 1.55 & 460 & 5.08 & 0.82 & 0.60 & 0.52 \\
\hline \multirow{2}{*}{10} & $0-30$ & 20.50 & 4.30 & 250 & 6.23 & 1.45 & 0.95 & 0.80 \\
\hline & $30-90$ & 15.00 & 3.10 & 60 & 3.34 & 0.70 & 0.63 & 0.59 \\
\hline \multirow{4}{*}{11} & $0-40$ & 15.00 & 3.40 & 50 & 3.21 & 0.74 & 0.92 & 0.70 \\
\hline & $40-70$ & 14.00 & 2.30 & 40 & 2.15 & 0.61 & 0.89 & 0.62 \\
\hline & $70-100$ & 10.00 & 1.40 & 50 & 2.05 & 0.50 & 0.51 & 0.40 \\
\hline & $100-150$ & 10.00 & 1.20 & 48 & 1.32 & 0.45 & 0.40 & 0.30 \\
\hline
\end{tabular}

\section{b. DTPA-extractable micronutrients:}

Data presented in Table (3) show that the values of chemically DTPAextractable $\mathrm{Fe}, \mathrm{Mn}, \mathrm{Zn}$ and $\mathrm{Cu}$ ranged 1.32-6.94, 0.45-2.14, 0.40-1.89 and 0.30$1.60 \mathrm{mg} \mathrm{kg}^{-1}$ soil. The highest values are found in the surface layer of soil profile No. 1 that represents soils developed on the Nile alluvium of Sahl El-Hussania. On the other hand, the lowest micronutrient contents are associated with the soils that are developed on the aeolian deposits that are siliceous in nature, i.e., poorer in nutrient-bearing minerals. Generally, the vertical distribution of DTPAextractable micronutrients reveals a tendency to accumulate in the surface layers, this behavior may be due to the additions of organic manures and agromanagement practices as well as the nature of soil sediments. These findings are in a good agreement with Ibrahim (2001).

According to the critical limits of DTPA-extractable $\mathrm{Fe}, \mathrm{Mn}, \mathrm{Zn}$ and $\mathrm{Cu}$, which have been reported by Soltanpour and Schwab (1977), their values ranged between Low \& Adequate limits. The corresponding limits were $(0-2 \&>$ $\left.4 \mathrm{mg} \mathrm{kg}^{-1}\right),\left(0-1.8 \&>4 \mathrm{mg} \mathrm{kg}^{-1}\right),\left(0-0.9 \&>1.5 \mathrm{mg} \mathrm{kg}^{-1}\right)$ and $(0-0.5 \&>0.5 \mathrm{mg}$ $\left.\mathrm{kg}^{-1}\right)$, respectively.

\section{Soil Taxonomy:}

Fayoum J. Agric. Res. \& Dev., Vol.24, No.2, July, 2010 
The guidelines of USDA System (1975) and updated Taxonomic Kev (2006) as well as soil morphological, physical and chemical data, which are presented in Table (1, 2, 3 and 4), were used for identifying soil taxa of the studied soils, which are developed on different parent materials and occupy widely portions of the east, west and south Nile Delta rims. The investigated areas are lying within a long hot rainless summer and short mild winter, with a pronounced amount of rainfall. Some soil profiles are enriched with secondary $\mathrm{CaCO}_{3}$ accumulations which satisfy the requirement of calcic horizon such as soil profile Nos. 5, 6 and 8 .

Table (4): Soil taxonomic units of the studied soil profiles.

\begin{tabular}{|c|c|c|c|c|c|}
\hline 它 & $\begin{array}{l}\dot{0} \\
\overline{0} \\
0 \\
1 \\
\dot{0} \\
\bar{Z}\end{array}$ & 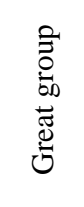 & $\begin{array}{l}\text { Sub- } \\
\text { group }\end{array}$ & Family & 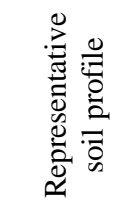 \\
\hline $\begin{array}{l}\infty \\
0 \\
0 \\
0 \\
0 \\
0\end{array}$ & 窇 & 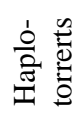 & 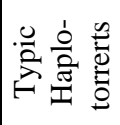 & Typic Haplotorrerts, fine clayey, smectitic, thermic & 1 and 2 \\
\hline \multirow{4}{*}{ 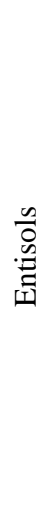 } & \multirow{2}{*}{$\underset{\stackrel{0}{0}}{\stackrel{0}{\Xi}}$} & \multirow{2}{*}{ 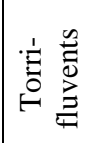 } & \multirow{2}{*}{ 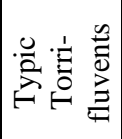 } & Typic Torrifluvents, sandy, mixed, thermic & 3 and 4 \\
\hline & & & & Typic Torrifluvents, clayey, smectitic, thermic & 9 \\
\hline & 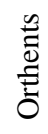 & 岳泀 & 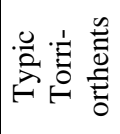 & Typic Torriorthents, sandy, mixed, thermic & 10 \\
\hline & 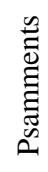 & 占泀 & 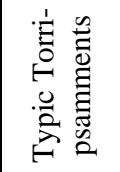 & Typic Torripsamments, siliceous, thermic & 7 and 11 \\
\hline $\begin{array}{l}0 \\
0 \\
0 \\
0 \\
0 \\
0\end{array}$ & $\frac{\sqrt[0]{0}}{\frac{0}{\tilde{n}}}$ & $\begin{array}{l}\frac{1}{2} \\
\frac{\pi}{\pi} \\
\frac{\pi}{\pi}\end{array}$ & 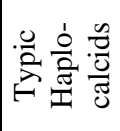 & Typic Haplocalcids, fine loamy, mixed, thermic & 5,6 and 8 \\
\hline
\end{tabular}

Accordingly, the studied soil profiles developed on the identified soil sites could be classified into three orders, i.e., Vertisols, Entisols and Aridisols, and six families, as follows:

i. Vertisols include one family of Typic Haplotorrerts, fine clayey, smectitic, thermic (i.e., soils of profile Nos. 1 and 2 that are developed on Sahl ElHussania).

ii. Entisols: include four families of Typic Torrifluvents, sandy, mixed, thermic (i.e., soils of profile Nos. 3 and 4 that are developed on El-Qurein and El-Salhia areas); Typic Torrifluvents, clayey, smectitic, thermic (i.e., soils of profile No. 9 that are developed on Tal El-Yahudia village); Typic Torriorthents, sandy, mixed, thermic (i.e., soils of profile No. 10 that are developed on El-Alikat El- 
Bahariya village) and Typic Torripsamments, siliceous, thermic (i.e., soils of profile Nos. 7 and 11 that are developed on El-Sadat and Abu-Zaabal areas).

iii. Aridisols: include one family of Typic Haplocalcids, fine loamy, mixed, thermic (i.e., soils of profile Nos. 5, 6 and 8 that are developed on El-Beheira and El-Nubaria areas).

V. Land suitability for irrigated agriculture soils:

a. Current land suitability:

The current suitability of the studied soils was estimated by matching between the present land characteristics and their ratings outlined by Sys and Verheye (1978) and Sys (1991). Suitability indices and evaluation of the studied soils developed on the studied different areas are shown in Table (5) and reveal that three suitability classes, i.e., marginally suitable (S3), moderately suitable (S2) and highly suitable (S1) were recognized in the studied areas. These classes represent some soils suffering from soil limitations, i.e., wetness (w), soil texture $\left(\mathrm{s}_{1}\right), \mathrm{CaCO}_{3}\left(\mathrm{~s}_{3}\right)$, gypsum $\left(\mathrm{s}_{4}\right)$ and salinity/alkalinity $(\mathrm{n})$, with different intensity degrees ranged between slight and severe. The corresponding limitation intensity degrees were slight, slight-severe, slight, slight and slight-severe, respectively.

Table (5): Land suitability classes for the studied soil profiles.

\begin{tabular}{|c|c|c|c|c|c|c|c|c|c|c|c|c|c|c|}
\hline \multirow[t]{2}{*}{$\stackrel{0}{0}$} & \multicolumn{2}{|c|}{$\begin{array}{c}\text { Topography } \\
\text { (t) }\end{array}$} & \multicolumn{2}{|c|}{$\begin{array}{l}\text { Wetness } \\
\text { (w) }\end{array}$} & \multicolumn{4}{|c|}{ Soil characteristics } & \multicolumn{2}{|c|}{$\begin{array}{c}\text { Salinity \& } \\
\text { alkalinity } \\
\text { (n) }\end{array}$} & \multicolumn{2}{|c|}{$\begin{array}{c}\text { Suitability } \\
\text { index } \\
(\mathrm{Ci})\end{array}$} & \multicolumn{2}{|c|}{$\begin{array}{c}\text { Suitability } \\
\text { class } \\
(\mathrm{Si}) \\
\end{array}$} \\
\hline & $\mathrm{Cs}$ & Ps & Cs & Ps & $\mathrm{S}_{1}$ & $\mathrm{~S}_{2}$ & $\mathrm{~S}_{3}$ & $\mathrm{~S}_{4}$ & Cs & Ps & $\mathrm{Cs}$ & Ps & $\mathrm{Cs}$ & Ps \\
\hline 1 & 100 & 100 & 80 & 100 & 85 & 100 & 95 & 90 & 50 & 100 & 29.70 & 72.67 & $\mathrm{~S} 3$ & $\mathrm{~S} 2$ \\
\hline 2 & 100 & 100 & 80 & 100 & 85 & 100 & 95 & 90 & 80 & 100 & 46.51 & 72.67 & $\mathrm{~S} 3$ & $\mathrm{~S} 2$ \\
\hline 3 & 100 & 100 & 90 & 100 & 85 & 100 & 85 & 90 & 100 & 100 & 58.52 & 62.02 & $\mathrm{~S} 2$ & $\mathrm{~S} 2$ \\
\hline 4 & 100 & 100 & 90 & 100 & 80 & 100 & 95 & 90 & 100 & 100 & 61.56 & 68.40 & $\mathrm{~S} 2$ & $\mathrm{~S} 2$ \\
\hline 5 & 100 & 100 & 100 & 100 & 95 & 100 & 90 & 90 & 100 & 100 & 76.95 & 76.95 & $\mathrm{~S} 1$ & $\mathrm{~S} 1$ \\
\hline 6 & 100 & 100 & 100 & 100 & 85 & 100 & 90 & 90 & 100 & 100 & 68.85 & 68.85 & $\mathrm{~S} 2$ & $\mathrm{~S} 2$ \\
\hline 7 & 100 & 100 & 100 & 100 & 40 & 90 & 95 & 90 & 100 & 100 & 34.20 & 34.20 & S3 & $\mathrm{S3}$ \\
\hline 8 & 100 & 100 & 90 & 100 & 95 & 100 & 90 & 90 & 90 & 100 & 62.33 & 76.95 & $\mathrm{~S} 2$ & $\mathrm{~S} 1$ \\
\hline 9 & 100 & 100 & 100 & 100 & 80 & 100 & 95 & 90 & 100 & 100 & 68.40 & 68.40 & $\mathrm{~S} 2$ & $\mathrm{~S} 2$ \\
\hline 10 & 100 & 100 & 90 & 100 & 45 & 100 & 95 & 90 & 98 & 100 & 33.93 & 38.47 & S3 & $\mathrm{S} 3$ \\
\hline 11 & 100 & 100 & 100 & 100 & 40 & 100 & 95 & 90 & 90 & 100 & 30.78 & 34.20 & S3 & $\mathrm{S} 3$ \\
\hline
\end{tabular}

$\mathrm{S}_{1}=$ Soil texture, $\mathrm{S}_{2}=$ Soil depth $(\mathrm{cm}), \mathrm{S}_{3}=$ Calcium carbonate status and $\mathrm{S}_{4}=$ Gypsum status

S3 = Marginally suitable, S2=Moderately suitable and S1=Highly suitable

$\mathrm{Cs}=$ Current land suitability and Ps=Potential land suitability

\section{b. Potential land suitability:}

Soil improvements practices are needed to correct or reduce the severity of soil limitations existing in the studied area for ameliorating its productivity, such as: a) Leveling of micro relief, b) Leaching of soluble salts that are existing in some of the studied soils, c) Construction of efficient open drainage ditches for lowering the saline water table, d) Continuous application of organic manure to improve soil physio-chemical properties and fertility status, e) Application of modern irrigation systems, i.e., drip and sprinkler in the newly reclaimed desert soils that are characterized by the relatively coarse textured grade to save a pronounced amount of irrigation water as well as to rise the irrigation efficiency and sustain soil moisture and available nutrients to uptake by plant roots.

By applying the aforementioned improvement practices, potential suitability of the studied soils indicate also the existing of three suitability classes of the current suitability, except some areas represented by soil profile Nos. 1, 2 and 8 that are shifted from S3, S3 and S2 to S2, S2 and S1, respectively. This is mainly due to their soil limitations that are able to correct, i.e., wetness and

Fayoum J. Agric. Res. \& Dev., Vol.24, No.2, July, 2010 
salinity/alkalinity conditions. It is noteworthy to mention that the similarity of suitability classes recognized in the studied area for both current and potential conditions are mainly attributed to most of the identified soil limitations are not able to correct. So, it could be recommended that the severity of soil texture (coarser in nature) can be corrected by application of organic and inorganic soil amendments as well as applying drip and sprinkler irrigation systems to sustain a soil moisture content at a favourable condition for grown plants and biological activity in the soil.

\section{REFERENCES:}

Black, C.A.; D.D. Evans; L.E. Ensminger; J.L. White and F.E. Clark 1965. Methods of soil analyses. Am. Soc. of Agron. Inc., Madison, Wisconsin, USA.

Gee, G.W. and J.W. Bauder 1986. Particle size analysis. In: Methods of Soils Analysi. Part I, Klute, A. (Ed.), Agronomy No. 9.

Gupta, I.C. 1990. Use of Saline Water in Agriculture. Publishing Co. Pvt Ltd, 66 Janpath, New Delhi, 110001.

FAO 1983. Keeping the land alive soil erosion, its causes and cures. FAO, Rome, Soil Bull. No. 50: 3-25.

FAO 1990. Guidelines for Soil Description. $3^{\text {rd. }}$ Ed. Revised, Soil Resources, Management and Conservation Service, Land and Water Development Division, Rome, Italy.

Follett, R.H. and W.L. Lindsay 1971. Changes in DTPA-extractable zinc, iron, manganese and copper in soils following fertilization. Soil Sci. Soc. Amer. Proc., 35: 600-607.

Ibrahim, Sh.B. 2001. Pedogenetic aspects as related to soil fertility status at El Fayoum, Egypt. Ph. D. Thesis, Fac. of Agric. at El Fayoum, Cairo Univ., Egypt.

Jackson, M.L. 1973. Soil Chemical Analysis. Prentice Hall of India Private Limited, New Delhi, India.

Lindsay, W.L. and W.A. Norvell 1978. Development of DTPA soil test for Zn, Fe, $\mathrm{Mn}$ and Cu. Soil Sci. Soc. Am. J., 42: 421.

Richards, L.A. 1954. Diagnosis and Improvement of Saline and Alkali Soils . U.S. Dep. of Agric. Hand book, No. 60, pp. 102.

Soltanpour, P.N. and A.B. Schwab 1977. A new soil test for simultaneous extraction of micronutrients in alkaline soils. Comm. Soil Sc. and Plant Annal., 8: 195.

Sys, C. 1991. Land evaluation . Parts I ,II and III , Lecture Notes. Ghent Univ., Ghent, Belgium.

Sys, C. and W. Verheye 1978. An attempt to the evaluation of physical land characteristics for irrigation according to the FAO Framework for land Evaluation. Int. Train. Cent. for Post Graduate Soil Scientists, Ghent Univ., Ghent, Belgium.

USDA 1975. Soil Taxonomy Basic System of Soil Classification. U.S. Dept., Agric. Handbook No. 436, USDA. Washington, D.C. 20402.

USDA 2006. Keys to Soil Taxonomy. $10^{\text {th }}(\mathrm{Ed})$, United States Department of Agriculture, Nature Resources Conservation Service.

Fayoum J. Agric. Res. \& Dev., Vol.24, No.2, July, 2010 


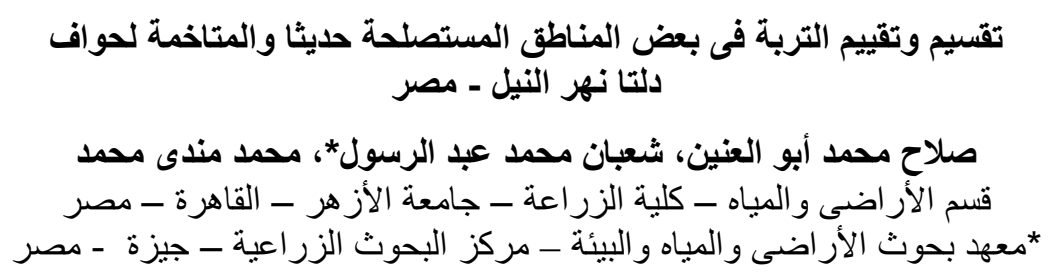

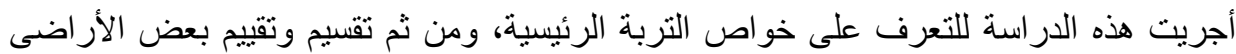

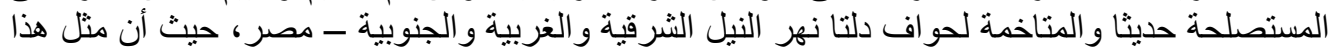

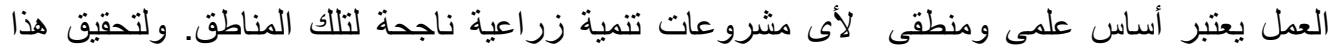

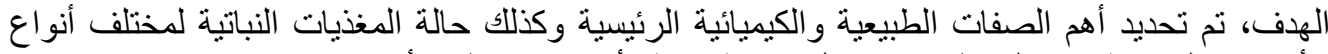

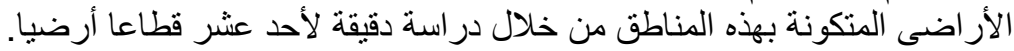

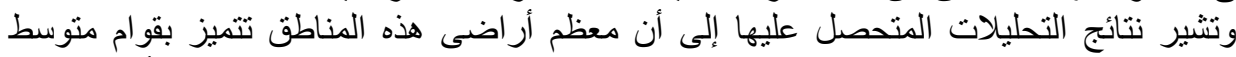

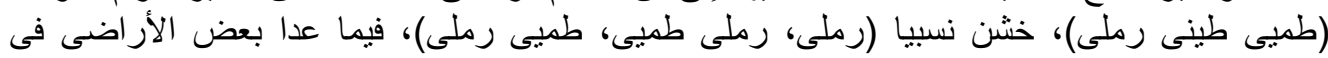

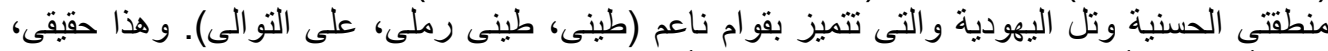

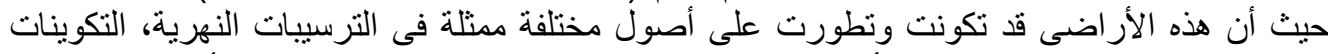

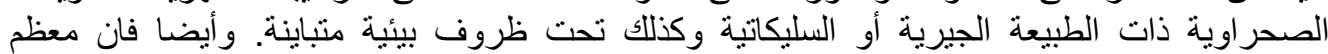

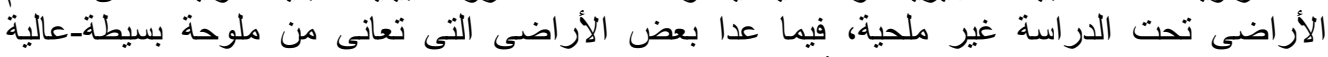

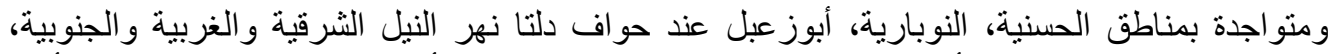

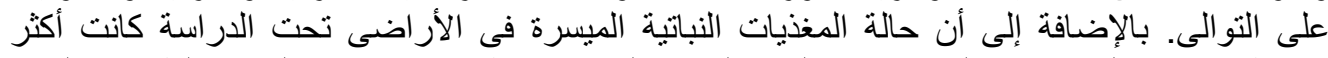
إرتباطا باضافة المخصبات العضوية، عمليات الخدمة الزراعية، طبيعة رسوبيات التربة التربة، الظروف البيائية

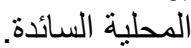

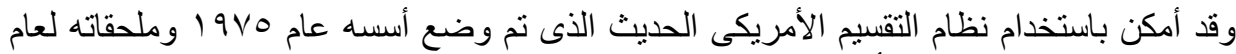

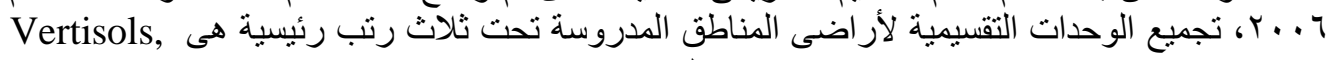
Entisols and Aridisols

i. Vertisols include one family of Typic Haplotorrerts, fine clayey, smectitic, thermic (i.e., soils developed on El-Hussania area).

ii. Entisols: include four families of Typic Torrifluvents, sandy, mixed, thermic (i.e., soils developed on El-Qurein and El-Salhia areas); Typic Torrifluvents, clayey, smectitic, thermic (i.e., soils developed on Tal El-Yahudia village); Typic Torriorthents, sandy, mixed, thermic (i.e., soils developed on El-Alikat El-Bahariya village) and Typic Torripsamments, siliceous, thermic (i.e., soils developed on El-Sadat and Abu-Zaabal areas).

iii. Aridisols: include one family of Typic Haplocalcids, fine loamy, mixed, thermic (i.e., soils developed on El-Beheira and El-Nubaria areas).

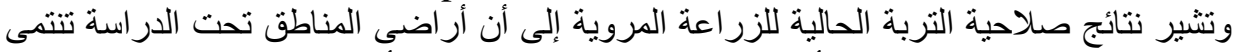

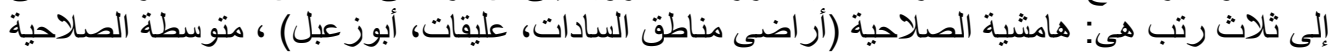

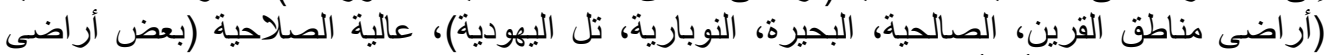

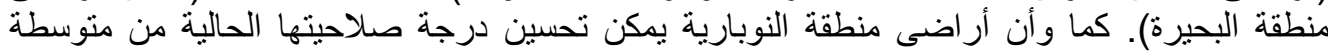
الصلاحية إلى عالية الصلاحية مستقبلا (الصلاحية الكامنة)، كنتيجة لقابلية معوقات الكات إنتاجية التربة ممثلة

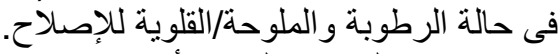

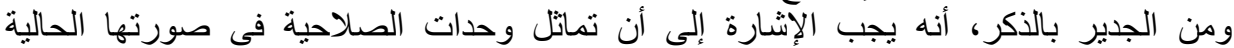

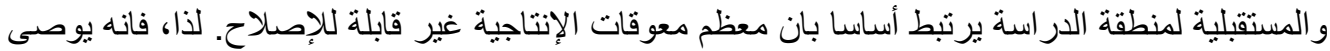

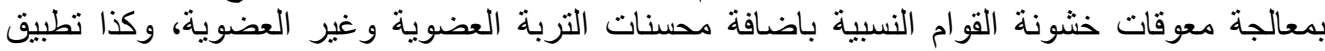
أنظمة الرى الحديثة (التنقيط و الرش) لتدعيم رطوبة التربة بالقدر المناسب للنباتية التبات النامية وتحفيز النشاط

$$
\begin{aligned}
& \text { الدحكين : أ.د. السيد عبدالحى خاطر أ.د. محمود خيرى } \\
& \text { البيولوجى فى التربة. }
\end{aligned}
$$

Fayoum J. Agric. Res. \& Dev., Vol.24, No.2, July, 2010 